\title{
An Automated Candling System for Duck Egg Fertility Detection, Sorting, and Counting via Digital Image Processing
}

\author{
Den Whilrex Garcia ${ }^{1+}$, and Glenn Magwili ${ }^{2}$ \\ ${ }^{12}$ Mapua University, Philippines
}

\begin{abstract}
Duck farming is considered one of the lucrative livelihoods in the Philippines because of its many advantages with minimal cost and high-profit returns. This is the second-largest poultry industry in the country, yet this industry is still immature and underdeveloped. There were many attempts done to improve the egg production sector of the poultry industry, particularly in the candling method. However, they mainly focused on chicken eggs. Hence, automating the candling system for the incubated duck eggs would be beneficial. The main objective of this study was to create an automated candling system for duck egg fertility detection, sorting, and counting via digital image processing. The hardware of the proposed system was made from locally available components, materials, and equipment. The most suitable light source was assessed, and it was found out that a 9W White LED would give the optimum results among the tested light bulbs. The camera was also calibrated and was placed three inches away from the incubated duck egg and was mounted ninety degrees from the horizontal. The fastest allocated processing time that would give a high fertility detection accuracy was assessed and was determined to be 2.5 seconds. The classification model was created in TensorFlow and was evaluated using a confusion matrix. The results showed that the classification accuracy was 0.86 , f-measure was 0.875 and MCC was 0.7417 . The functionality was also assessed and was $100 \%$ functional. The accuracy of the machine to detect the fertility of 5-, 6-, 7-, 8-, 9- and 10-day old, incubated duck eggs were also tested and were found out to be $50 \%, 60 \%, 90 \%, 100 \%, 80 \%$, and $80 \%$ accurate, respectively. The counting of total inputted duck eggs and total sorted "balut" and "penoy" was also $100 \%$ accurate. Lastly, the efficiency of the system was evaluated by calculating the overall equipment effectiveness and was found out to be $83.99 \%$ efficient.
\end{abstract}

Keywords: automation, agriculture, duck egg candling, artificial neural network, digital image processing

\section{Introduction}

The act of making and eating fertilized duck eggs, which is locally known as "balut" in the Philippines, is a widely known practice in Asia [1], because of its extraordinary taste and nutritive value [2]. According to [3] during the 4 th quarter of 2019 , there was a recorded uptrend in the production volume of duck eggs amounting to a $20.4 \%$ increase due to a higher demand for "balut" followed by salted eggs or "itlog na maalat". This report strengthens the circumstance that duck farming is deemed as a lucrative livelihood in many Asian countries [4], including the Philippines. As a substantial fact, the "balut" industry has been established a long time ago in the country, yet even now, stays immature and underdeveloped [1]. As stated in the paper of [5], one of the crucial factors in alleviating the productivity of the egg industry is to ensure that the eggs placed in the incubators are fertilized. This will enhance the productivity of eggs, boost egg fertility rate, give more space, and reduce incubation and labour costs. Herewith, fertility detection must be performed, and this is done thru the candling process. However, the current procedure in small-scale and backyard farms is labour extensive, time-consuming, and is susceptible to human errors because of overtiredness and sight errors of workers who must examine thousands of eggs per day. Some of the recent various machine vision system for egg fertility detection includes [5-8]. All focused primarily on chicken egg fertility detection and were proven of tantamount help to the chicken egg industry. This can also be beneficial when implemented to duck eggs. Furthermore, their system did not include the counting process, which is also time-consuming and is prone to human errors. Hence, the development of a vision system for duck egg fertility detection, sorting, and counting would be valuable to small-scale and backyard farms. The primary objective of this study was to develop an automated candling system for the detection, sorting, and

\footnotetext{
+ Corresponding author. Tel.: + 63464040163

E-mail address: whilrex@gmail.com
} 
counting of fertilized and unfertilized duck eggs via digital image processing. The specific objectives of the study were: (1) to develop and to fabricate the hardware of the automated candling system that can handle duck eggs for detection, sorting, and counting; (2) to apply artificial neural network and digital image processing as the algorithm for the duck egg fertility detection and to develop the program of the entire system in detecting, sorting, and counting fertilized and unfertilized duck eggs; and (3) to test and to evaluate the system's functionality, accuracy, and efficiency. The general scope of the study was the development of an automated candling system for the detection, sorting, and counting of fertilized and unfertilized incubated duck eggs. The study focused on capturing images of duck eggs using the camera module, processing the image via the microcomputer, classifying the fertility of the eggs, sorting, and counting them, and displaying the data through a monitor display. The study was limited only to the identification and sorting of fertilized duck eggs (balut) and unfertilized duck eggs (penoy) happening on the first candling. The system would never recognize other parameters aside from the pattern of the eggs' embryo, and the colour of the egg after illumination. The system could not detect the presence of cracks, nor the egg's rottenness. Furthermore, the eggs would be placed manually onto the conveyor of the prototype and were also manually removed after sorting and counting. The developed machine was tested and evaluated at Restynor Balut Industry of General Trias City, Cavite, Philippines.

\section{Related Studies and Literature}

\subsection{Local Process of "Balut" Making}

Fertilized duck eggs, locally known as "balut", is an authentic Filipino delicacy [1] made from incubated eggs of Philippine Mallard ducks, notably known as "Pateros Itik". The process of making "balut" starts with a "pitik system" to ensure that thick-shelled duck eggs will be the ones to be incubated, and not the thinshelled or the ones with cracks [9]. This is done because thick-shelled eggs have the capability to handle the dreary process of incubation. The thin-shelled and cracked eggs will be sold as either raw eggs or salted eggs. Next, the eggs are sun-dried for 2 to 7 hours to provide warmth for the eggs [10] and to remove any residual moisture on the eggs. Then after this, the 16- to 18-days incubation takes place. However, on the 10th day of incubation, candling will be done to determine whether the incubated egg will be sold as "penoy" or "balut". In this procedure, eggs are quickly removed from the incubator, and each is allowed to pass from a lighted device called "silawan" [11]. With this device, the balut maker or "magbabalut" can determine whether to sell the egg as "balut" or not. If the egg shows a spider-like structure with a dark spot in the middle when illuminated, then the egg is fertilized, and it will be brought back into the incubator. Otherwise, if the egg shows transparency and appears yellow orangish, then the egg is unfertilized, and it will be sold as "penoy". Eggs with dead embryos are also considered as "penoy" and are not returned to the incubator. After the incubation period, the eggs will be candled for the second time to ensure that the embryos are alive. Eggs with normal embryos will be air-dried momentarily, then will be boiled for 20 to 30 minutes and finally, will be sold as "balut" [12].

Candling is therefore substantial to check the fertility of incubated duck eggs. This is also done to prevent contaminating fertilized duck eggs due to the detonation of unfertilized ones [13]. This method is also a non-destructive process and must be done in a totally dark room. In small-scale and backyard farms, candling is usually done manually by a worker or operator, who relies solely on his/her knowledge, skills, and experience. Thus, this process is susceptible to human errors and mistakes [8]. In addition, since this manual method is done in a dark room for several hours a day, the operator gradually damages his/her eye vision and develops other health issues including headaches, musculoskeletal disorders, stress, and burnouts [8].

\subsection{Various Machine Vision System}

From the previous years, there were numerous machine vision systems proposed by local and foreign authors due to their many advantages. These include the work of [14-17]. The promising results from these studies benefitted the progress and the development of this study. Calculation of hue and saturation of each RGB pixel was adopted as the principal filtering parameters in the study since duck egg fertility detection via candling is also a colour-based categorisation process. 
Several studies attempted to improve the egg fertility detection, specifically the candling process, of various avian. In [6], detection of chicken egg fertility using image processing techniques was developed with $85 \%$ accuracy. In [5], a machine vision system for detecting fertilized chicken eggs was also developed with high accuracy however, their machine should be in a totally dark room which is not advisable for labourers due to health risks. In [7], their proposed method is via measuring oxygen flux of incubated eggs, however, this is a complex method and is not practical in small-scale and backyard farms. In [8], a merged manual and automatic candling system were developed to solve the social issues in their country. In [18], a new low-cost detection device for early discrimination of eggs fertility using an advanced statistical classifier was built, however, the downside is that their work is not yet fully automated, and the accuracy of detection is based on the position of the sensor. All focused predominantly on chicken eggs and not on duck eggs, wherein in the Philippines, the duck egg industry is considered a profitable livelihood. Thus, having an automated candling machine vision system for duck egg fertility detection, sorting, and counting would be of great help to the industry.

\section{Methodology}

The study was an experimental setup and concentrated on the development of the algorithm and prototype for the automated candling, sorting, and counting machine for duck eggs. The whole methodology was divided into four phases, which were comprised of ten various research steps. Phase 1 was dedicated to gathering information, analysis, and synthesis. This aided the researcher to have a deeper understanding and strong foundation about the topic. Phase 2 was dedicated to the development and fabrication of the mechanical body, including the electronic part, of the prototype. A fully functional hardware prototype was the output of the step and served as the product of the entire Phase 2. Phase 3 was dedicated to the algorithm development for the artificial neural network and digital image processing, and the program development for the entire system. A fully functional prototype, that was ready for evaluation was the output of this phase. The last phase was the evaluation of the complete prototype's ability to detect, sort, and count fertilized and unfertilized duck eggs. At the end of this phase, the researcher was able to verify and conclude the functionality, accuracy, and efficiency of the system. Fig. 1 presents the accomplished methodology.

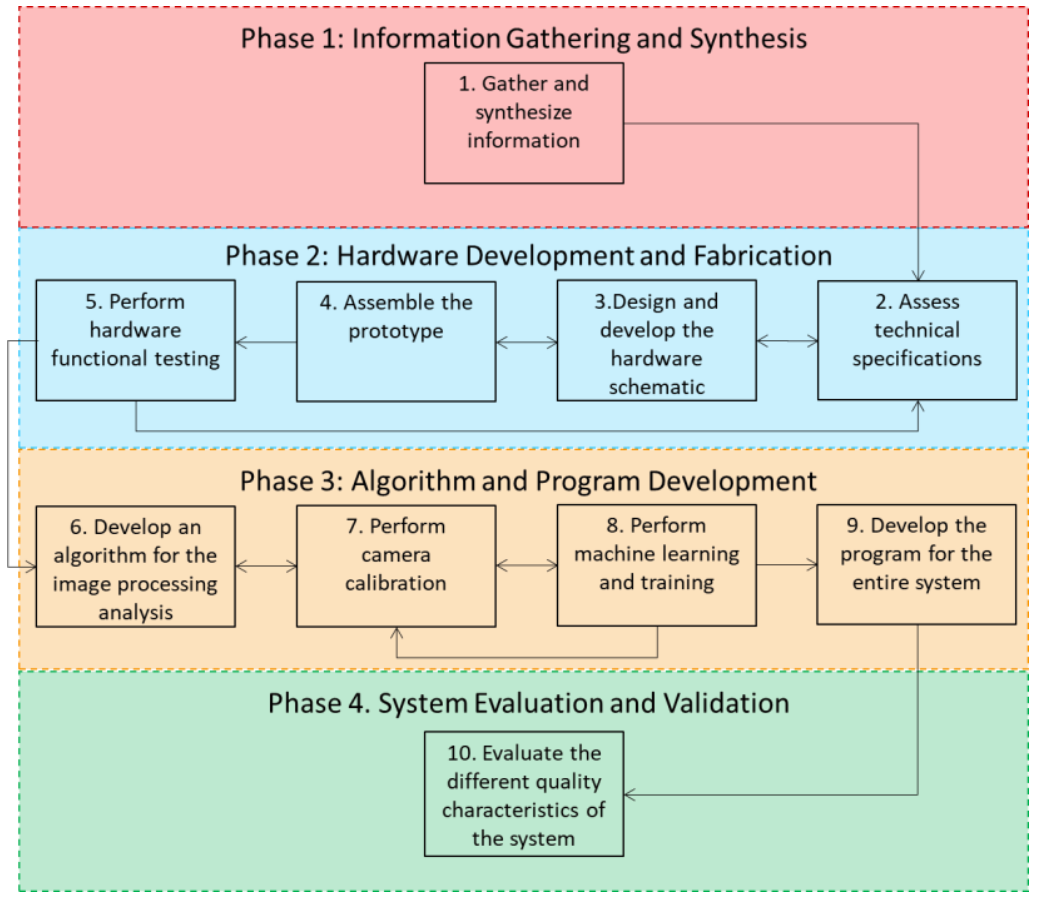

Fig. 1: The Implemented Four Phases and Ten Research Steps to Complete the Study

\section{Results}

The prototype was made using locally available components, materials, and equipment. The major components include a stepper motor, which moved the conveyor system of the machine; two servo motors, 
which were used to push the incubated duck eggs after detection to the proper egg containers; a motor driver, which acted as the interface between the microcontroller and the servo motors; five proximity sensors; an LCD monitor; a buzzer; a raspberry pi microcomputer; a raspberry pi camera module, and; an Arduino microcontroller board.

The fabricated hardware of the developed prototype was divided into three sections: (1) the egg transporter section, (2) the egg candling chamber section, and (3) the egg sorter section as in Fig. 2a. The egg transporter section is where the eggs were being placed manually on the holder, one by one, and was then being transferred to the egg candling chamber section. The next section is where the eggs were being analysed and categorised as either fertilized duck egg (balut) or unfertilized duck egg (penoy) and were then being moved to the egg sorter section. The last section is where the eggs were being sorted and were being moved to the correct egg containers. There were two containers, the left is for "balut", while the right one is for "penoy". Fig. 2b presents the inside of the egg candling chamber. (1) is where the camera was mounted; (2) is where the eggs were being positioned for image capturing and for fertility detection; (3) is where the proximity sensor was placed, and; (4) is where the light source was located.
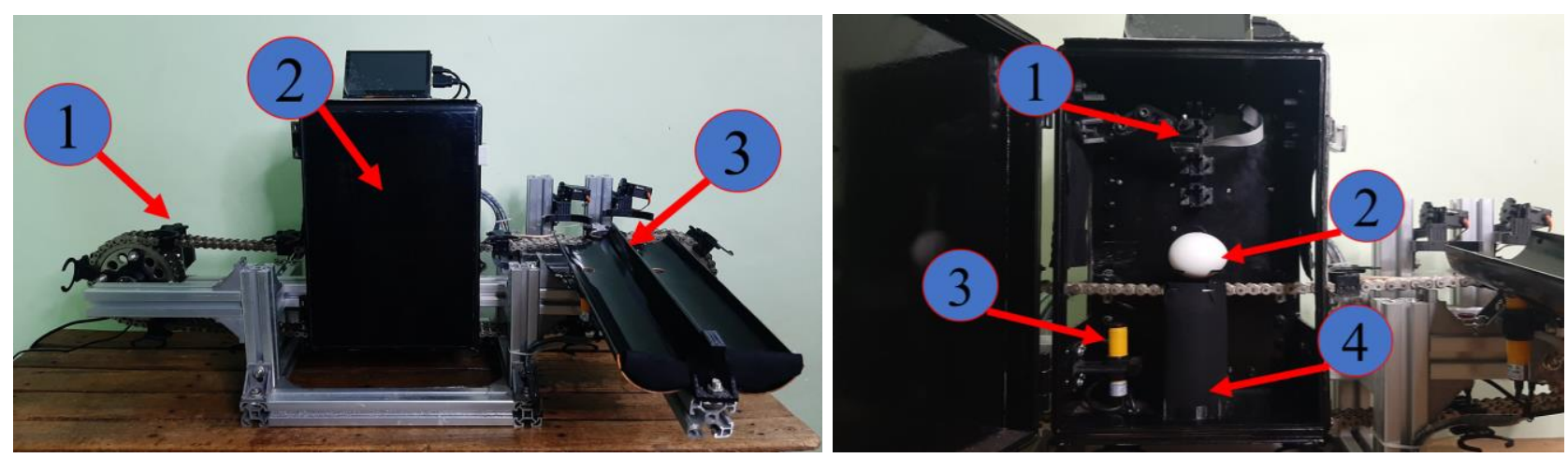

Fig. 2: Photo of the Actual Developed Prototype: (a) the Front View of the Entire System; (b) the Interior of the Egg Candling Chamber Section

The graphical user interface (GUI) of the system was being displayed by an LCD monitor located at the top of the egg candling chamber. This displayed what the camera sees, the fertility of the incubated duck egg being classified, the start and reset button, and the total number of inputted and sorted duck eggs. Fig. 3a shows what was being displayed whenever the program is initializing while Fig . $3 \mathrm{~b}$ shows what can be seen in the GUI once initialisation was done.
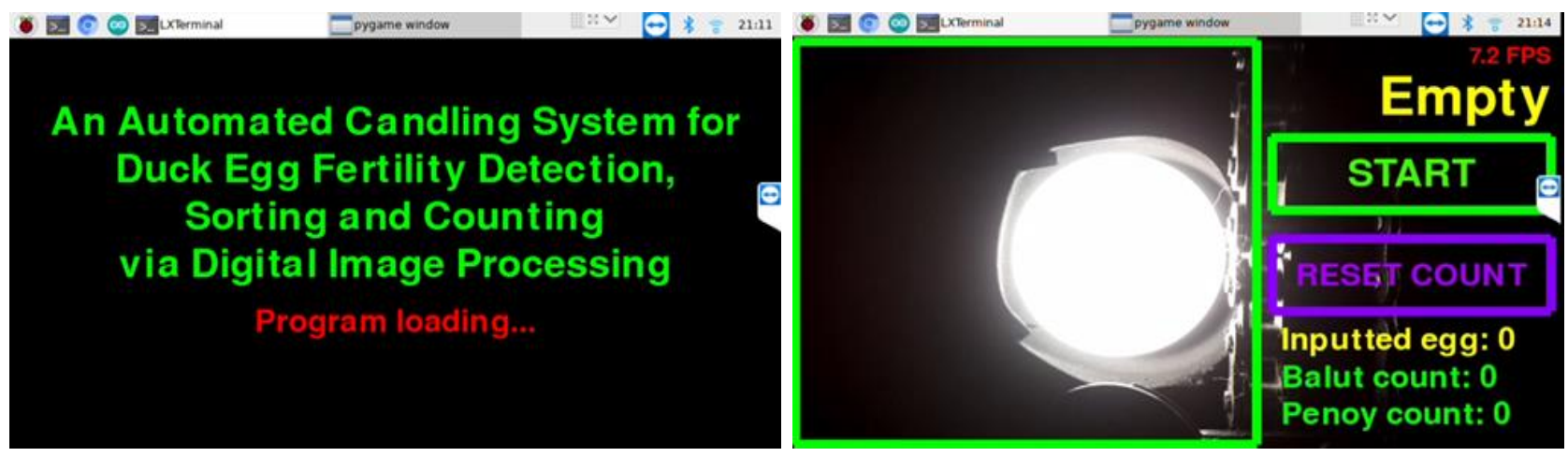

Fig. 3: Photo of the GUI: (a) When the Program is Initializing; (b) When the Program is Done with Initialization

The first alpha testing was about the detection of the best light source that is locally available in the market. Fig. 3 shows the result. The light sources that were assessed, starting from the leftmost to the rightmost, include a 5W White Light Emitting Diode (LED), a 7W White LED, a 9W White LED, a 7W Yellow LED, an 8W compact fluorescent light (CFL) bulb, an 11W CFL bulb, and a 25W incandescent bulb. 
The top images were fertilized duck eggs while the bottom images were unfertilized ones. Through this, it was found out that the $9 \mathrm{~W}$ White LED illuminates the inside of incubated egg the clearest.
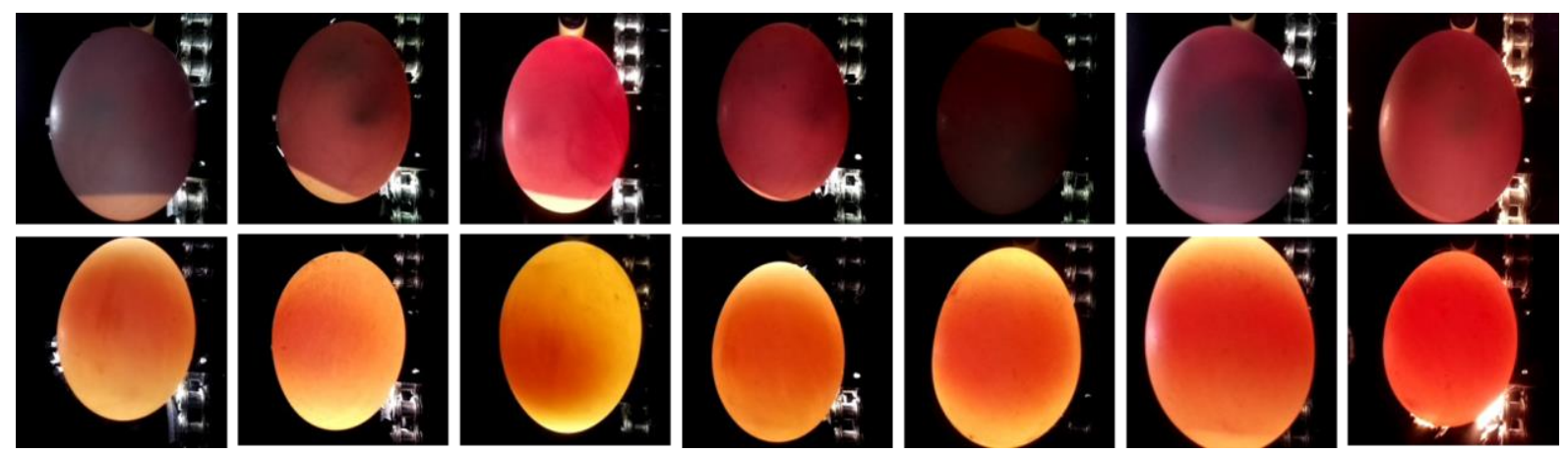

Fig. 4: Sample Captured Image Using Different Light Sources

The second alpha testing was dedicated to camera calibration. The proper distance of the camera to the incubated duck egg and the proper angle of the camera from the horizontal was tested. For both testings, each parameter was tested in 3 trials and each trial was tested 10 times using pre-detected duck eggs by an experienced candler. For the distance test, 2 inches until 5 inches were tested, with a 1-inch increment per testing. Through this, it was found out that the most appropriate was 3 inches. For the angle test, 60 degrees until 120 degrees were tested, with a 15-degree increment per testing. And for this, it was found out that the most appropriate was 90 degrees from the horizontal.

The third alpha testing was dedicated to the determination of the fastest processing time that will give a high accuracy of detection. Like the previous alpha testing, each processing time was tested 3 trials and each trial was tested 10 times. Starting from 0.5 seconds until 5.0 seconds, with a 0.5 -second increment per testing. And for this, the fastest processing time that gave a high fertility detection accuracy was 2.5 seconds.

For the creation of the classification model, transfer learning was used for the artificial neural network. A pre-trained neural network was used, and the last layer of the neural net was the classes. The classes include the set of images for empty egg holders, the set of images for fertilized incubated eggs, and the set of images for unfertilized duck eggs. The Keras model was built in TensorFlow. A total of 1000 images were gathered for fertilized duck eggs, 300 for unfertilized duck eggs, and 300 for empty holders. The dataset was divided into $75 \%$ training dataset and $25 \%$ validation dataset. A confusion matrix was created to summarize the prediction results of the duck egg fertility detection, by recording the number of correct and incorrect predictions with count values and broken down by each class. This gave insights into not only the errors being made by the developed duck egg fertility detection system but also the types of errors that were being made by the system. The true positive (TP) was the number of fertilized eggs classified by the system as indeed fertilized; false negative (FN) was the number of fertilized eggs classified as unfertilized; true negative (TN) was the number of unfertilized eggs classified as unfertilized, and; false positive (FP) was the number of unfertilized egg classified as fertilized. Then the classification accuracy, f-measure, and Matthews correlation coefficient (MCC) were calculated to assess the accuracy of the system to detect duck egg fertility. With all these measures, the best value is 1.0. Table 1 shows the created confusion matrix.

Table 1: Confusion matrix for the developed classification model

\begin{tabular}{|l|l|l|l|}
\hline $\mathrm{n}=100$ & Classified as Fertilized Duck Egg & Classified as Unfertilized Duck Egg & Total \\
\hline Actual Fertilized Duck Egg & $\mathrm{TP}=49$ & FN $=1$ & 50 \\
\hline Actual Unfertilized Duck Egg & $\mathrm{FP}=13$ & $\mathrm{TN}=37$ & 50 \\
\hline Total & 62 & 38 & 100 \\
\hline
\end{tabular}

Classification Accuracy $=(\mathrm{TP}+\mathrm{TN}) /(\mathrm{TP}+\mathrm{TN}+\mathrm{FP}+\mathrm{FN})$

$$
\text { Recall }=\mathrm{TP} /(\mathrm{TP}+\mathrm{FN})
$$




$$
\text { Precision }=\mathrm{TP} /(\mathrm{TP}+\mathrm{FP})
$$

F-measure $=(2 \times$ Recall $\times$ Precision $) /($ Recall + Precision $)$

$$
\mathrm{MCC}=(\mathrm{TP} \times \mathrm{TN}-\mathrm{FP} \times \mathrm{FN}) / \operatorname{sqrt}((\mathrm{TP}+\mathrm{FP}) \times(\mathrm{TP}+\mathrm{FN}) \times(\mathrm{TN}+\mathrm{FP}) \times(\mathrm{TN}+\mathrm{FN}))
$$

The classification accuracy was calculated to be 0.86 . The recall and precision were computed as 0.98 and 0.7903 , respectively. These values were used to get the f-measure, which uses the harmonic mean instead of the arithmetic mean. F-measure was calculated as 0.875 . Lastly, MCC was calculated as 0.7417.

The final evaluation includes the functionality, accuracy, and efficiency testing.

The functionality was tested to verify the ability of the prototype to perform its essential purpose and to execute each specific function that it is capable of, via having a checklist of all the anticipated distinct functions. The functionality testing included running and assessing the prototype, as a whole, in terms of its performance at various conditions, starting from powering on the device until shutting it down. Each function was tested in five trials. It was found out that the developed machine was $100 \%$ functional.

The accuracy of the system was assessed to evaluate its state of being correct and precise in detecting, sorting, and counting fertilized and unfertilized duck eggs. This was measured by assessing the unknown fertility of a set of ten incubated duck eggs using the manual candling and the developed system. There were six sets, and each set corresponds to different ages of duck eggs. Set 1 was for 10-day old eggs; set 2 was for 9-day old eggs; set 3 was for 8-day old eggs; set 4 was for 7-day old eggs; set 5 was for 6-day old eggs and; set 6 was for 5-day old eggs. Each egg in each set was marked with its corresponding test subject number and was incubated until the expected maturity date. On the 17th day inside the incubator, the eggs were candled again to verify fertility. Table 2 shows the comparison of the two methods. With this, the accuracy test in the number of correct detections of incubated eggs in manual and developed prototype using paired $\mathrm{t}-$ test was found out to be no significant difference at 0.05 level. Therefore, the automation of the candling system was successful. Furthermore, the accuracy was also measured in terms of the correctness of the LCD monitor in presenting the total count of inputted duck eggs and sorted "penoy" and "balut" using another six sets of eggs with five "balut" and five "penoy". It was found out that the counting of total inputted egg and total sorted eggs was $100 \%$ accurate.

Table 2: Accuracy Test in the Number of Correct Detections of Incubated Eggs in Manual and Prototype Using

\begin{tabular}{|c|c|c|}
\hline Number of Days in Incubator & Percent of Correct Detection (in Manual) & Percent of Correct Detection (in Prototype) \\
\hline 5 & 40 & 50 \\
\hline 6 & 60 & 60 \\
\hline 7 & 70 & 90 \\
\hline 8 & 100 & 100 \\
\hline 9 & 90 & 80 \\
\hline 10 & 90 & 80 \\
\hline Mean & 75.00 & 76.67 \\
\hline Std. Deviation & 22.583 & 18.619 \\
\hline Mean Difference & \multicolumn{2}{|c|}{1.67} \\
\hline$t$ - Value & \multicolumn{2}{|c|}{0.057} \\
\hline$d f$ & \multicolumn{2}{|r|}{5} \\
\hline$p$-Value & \multicolumn{2}{|c|}{0.957} \\
\hline Interpretation & \multicolumn{2}{|c|}{ No Significant Difference at 0.05 level. } \\
\hline
\end{tabular}
Paired t-Test

The efficiency of the system was measured and evaluated by calculating the overall equipment effectiveness (OEE) as in [19]. This was considered a straightforward and convenient yet formidable 
calculation tool because this takes the most common sources of productivity losses and divided them into three understandable categories [20]. These categories comprise availability, performance, and quality.

$$
\mathrm{OEE}=\text { Availability } \times \text { Performance } \times \text { Quality }
$$

First, availability was determined. Availability takes into account all the time it took to stop the production itself, which includes, break time of the candlers, changeover time, and machine downtime. For every 4-hour shift of a candler at Restynor Balut Industry, the break time lasts for a maximum of fifteen minutes. Changeover time lasts for five minutes, and a machine downtime was assumed to be, at maximum, fifteen minutes. Thus, the overall downtime per shift was thirty-five minutes. Therefore, availability was calculated as $85.42 \%$. Next, for the performance, the average time it took from detecting the fertility of incubated duck eggs to sorting and counting using the proposed system was six seconds. Thus, for one minute, the machine could sort ten eggs. So, for the entire four-hour shift, the machine could sort 2400 eggs. However, since the speed of the motors was fixed, the same as the processing time of the captured images, there would be no instance that the machine cycle would be slower. Therefore, it was found out that the performance was $100 \%$. Lastly, for the quality, six sets of eggs, where each set contains ten eggs, were evaluated. This category of OEE tested whether the eggs would be sorted by the machine safely and without cracking or making the egg fell from the egg holder. With the sixty eggs, one got fell from the holder. Assuming that in every sixty eggs, there would be at least one egg that would be cracked, therefore, out of 2400 eggs, there would be forty cracked eggs. Thus, quality was calculated as $98.33 \%$. Therefore, OEE was calculated as $83.99 \%$.

\section{Conclusion}

Based on the information, observations and results gathered, the automated candling system for duck egg fertility detection, sorting, and counting via digital image processing met the target objectives.

The hardware of the automated candling system was successfully and efficaciously developed and fabricated. The three sections of the system, namely, the egg transporter section, the egg candling chamber section, and the egg sorter section were fully operational. The distance of the camera from the incubated duck egg being categorised, the angle of orientation of the camera from the horizontal, and the decision time for the microcomputer that will give the optimum results were assessed successfully.

The artificial neural network and digital image processing were successfully developed as the algorithm to detect the fertility of incubated duck eggs. The developed classification model was assessed using a confusion matrix and the calculations of different parameters showed that the model was of good performance. The entire program for the system was also excellently developed.

The device was also tested and was evaluated through technical evaluations. The functionality of the machine was assessed and was found out to be $100 \%$ functional. The accuracy in detecting different ages of incubated duck eggs was also assessed. It was found out that the machine has good performance in classifying the fertility of 10-, 9-, and 8-day old duck eggs. It also has acceptable performance for 7-, 6-, and 5-day old eggs and could detect better as compared with the existing manual candling method. The overall equipment effectiveness was found out to be $83.99 \%$. Overall, the proposed machine met the desired objectives.

\section{Acknowledgment}

The authors would like to thank Mapua University, especially the School of Graduate Studies for the untiring support and unceasing guidance from the very beginning until the completion of the research. They also want to extend their sincerest gratitude to the Engineering Research and Development for Technology (ERDT) under the Department of Science and Technology (DOST) for funding this study.

\section{References}

[1] M.C.P. Alejandria, T.I.M. De Vergara, and K.P.M. Colmenar. The authentic balut: history, culture, and economy 
of a Philippine food icon. J. Ethn. Food 6, 16 (2019). https://doi.org/10.1186/s42779-019-0020-8

[2] H. S, Chang, C. Dagaas, N. de Castro, R. Ranola, A. Lambio, and M. L. Malabayuabas. An overview of the Philippine duck industry. 2003 Conference (47th), February 12-14, 2003, Fremantle, Australia 57842, Australian Agricultural and Resource Economics Society.

[3] Philippine Statistics Authority. Performance of Philippine agriculture fourth quarter 2019 (October to December 2019). Republic of the Philippines: Philippine Statistics Authority, 2020

[4] B. Jha and C. Chakrabarti. Duck farming: a potential source of livelihood in tribal village. Journal of Animal Health and Production. 2017, 5, 2:39-43.

[5] M. Hashemzadeh, and N. Farajzadeh. A machine vision system for detecting fertile eggs in the incubation industry. 2016. 9,5: 850-892.

[6] M. Bhuvaneshwari, and L.M. Palanivelu. Improvement in detection of chicken egg fertility using image processing techniques. International Journal on Engineering Technology and Sciences. 2, 4: 64-67. Modelling Simul. Mater. Sci. Eng. 2015, 13 (3): 173-183.

[7] W. Qiaohua, F. Dandan, M. Meihu, M and T. Zhang. Differentiating between fertilized and unfertilized eggs prior to incubation based on oxygen flux measurement. Int J Agric \& Biol Eng. 2017. 10,4: 243-251

[8] Vargas Cruz Ramiro Sebastián, Ruiz Salvador Lourdes Cecilia, Navas Lema María Cristina. Merging manual and automated egg candling: a safety and social solution. Enfoque Ute Revista, 9 (No.2). 2018. Retrieved from http://oaji.net/articles/2017/1783-1530283614.pdf

[9] N. Sanceda, K. Ueda, J. Ibenez, E. Suzuki, M. Kasai, and K. Hatae. Some fine aspects boiled and historical fertilized background and of "balut" and 'penoy', boiled incubated fertilized and unfertilized duck eggs. J Cookery Sci Japan. 2007. 40:231-8.

[10] T. Matejowsky. The incredible, edible balut. Food Culture Society. 2013. 16:387-404.

[11] R. Escobin, T. Medialdia, and C.F. Caramihan. Quality of 'balut' (fertilized duck egg) produced in four rice-duck zones of Laguna. Philippine. J Vet Animal Sci. 2009:35.

[12] M. Magat Balut: fertilized duck eggs and their role in Filipino culture. Western Folklore. 2002. 61:63-96. https://doi.org/10.2307/1500289.

[13] T. Daniels, T. Candling Eggs. 2018. https://poultrykeeper.com/incubating-and-hatching-eggs/candling-eggs/ Retrieved February 10, 2020.

[14] A. H. Jr. Ballado, J.C. Dela Cruz, G.O. Avendaño, N.M. Echano, J.E. Ella, M.E.M. Medina, and, B.K.C. Paquiz. Philippine currency paper bill counterfeit detection through image processing using canny edge technology. 8th IEEE International Conference Humanoid, Nanotechnology, Information Technology Communication and Control, Environment and Management (HNICEM) 2015. Conference Proceedings

[15] A.H. Jr. Ballado, L.C. David. Mapping mangrove forest from LiDAR data using object-based image analysis and support vector machine: The case of Calatagan, Batangas. 8th IEEE International Conference on Humanoid, Nanotechnology, Information Technology, Communication and Control, Environment, and Management, HNICEM. 2015. Conference Proceedings

[16] A. Wilson. Sorting color-coded cubes. Vision Systems Design. https://www.vision-systems.com/camerasaccessories/article/16738561/sorting-colorcoded-cubes. Retrieved February 10, 2020.

[17] F. Taqi, F. Al-Langawi, H. Abdulraheem, and M. El-Abd. A cherry-tomato harvesting robot. 18th International Conference on Advanced Robotics (ICAR), Hong Kong, 2017, pp. 463-468.

[18] S.E. Abdallah, W.M. Elmessery, and A.A. Elseify, A new low-cost detection device for early discrimination of eggs fertility using advanced statistical classifiers. Misr. J. Ag. Eng. 2018. 35(1): 199-226

[19] Subramaniam, S. \& Husin, Siti \& Yusop, Yusmarnita \& Hamidon, A.. Machine efficiency and man power utilization on production lines. 2008. 40-45.

[20] P. Godfrey. Overall equipment effectiveness. IEEE Transactions on Semiconductor Manufacturing 2002, Vume 18, Issue 1, Page(s): 190 - 196 